\title{
Enterprise
}

\section{Robotic Process}

\section{Automation}

Robotic Process Automation (RPA) is an emerging technology widely used across multiple sectors such as human resources, healthcare, finance, accounting, manufacturing, higher education and supply chain management, etc. RPA, also known as 'software bots', replaces manual, rule-based, repetitive tasks humans perform. These software bots are currently in a journey, evolving to be more sophisticated, mimicking human activities and enabling humans to achieve higher-valued tasks. Hence, RPA impacts the overall operational efficiency in organisations through multiple facets by its integration with employees, existing technologies and infrastructure, and business processes. It reduces the burden on IT as it does not disturb the underlying legacy systems. It increases reliability as bots can work $24 \times 7$ effectively. It is used as a time and cost reduction technology as it reduces the size of the manual workload. The tasks performed through RPA is accurate as it is less prone to errors. It increases compliances as it follows the rules and keeps audit trails. The productivity rate of organisations increase as the execution time through RPA is faster than tasks being performed by human employees. Furthermore, RPA is introduced as a low code technology that uses drag and drop functionalities with little to no programming knowledge [1].

Organisations begin the RPA implementation after a readiness and a feasibility study. The first stage of any RPA implementation is the "Proof of concept $(\mathrm{PoC})$ ", and most organisations are currently in their PoC stage of the RPA journey. However, Gartner predicts that RPA is on its way towards becom- ing a mainstream technology and will disrupt the market in the recent years to come. RPA is usually surrounded by scepticism as a technology that will steal human jobs, a mere automation strategy hyped by vendors and service providers, a short-term solution to organisational efficiency issues. The lack of case studies and business cases publicly available, discussing the benefits and disbenefits of RPA is also a reason for this scepticism.

Nevertheless, Garner states that RPA has already proven its value by delivering significant returns on investments in short payback periods. RPA is predicted to be deployed on a large scale in the future. With the steady maturity of the RPA market, different toolboxes such as maturity frameworks, readiness frameworks, etc., for RPA have also come into play at global levels. This large-scale deployment leads to the new field of study, 'The enterprise-wide RPA.'

\section{What is Enterprise RPA?}

Research has investigated that most organisations implementing RPA is at its Proof of Concept or Pilot phase and are experiencing difficulties scaling up [2]. The main reason for the struggle in scaling up is that RPA's implementation approach as a new technology is different from implementing other technologies. RPA is rapid automation that must be embedded into the organisation with high involvement of business area, IT department and 


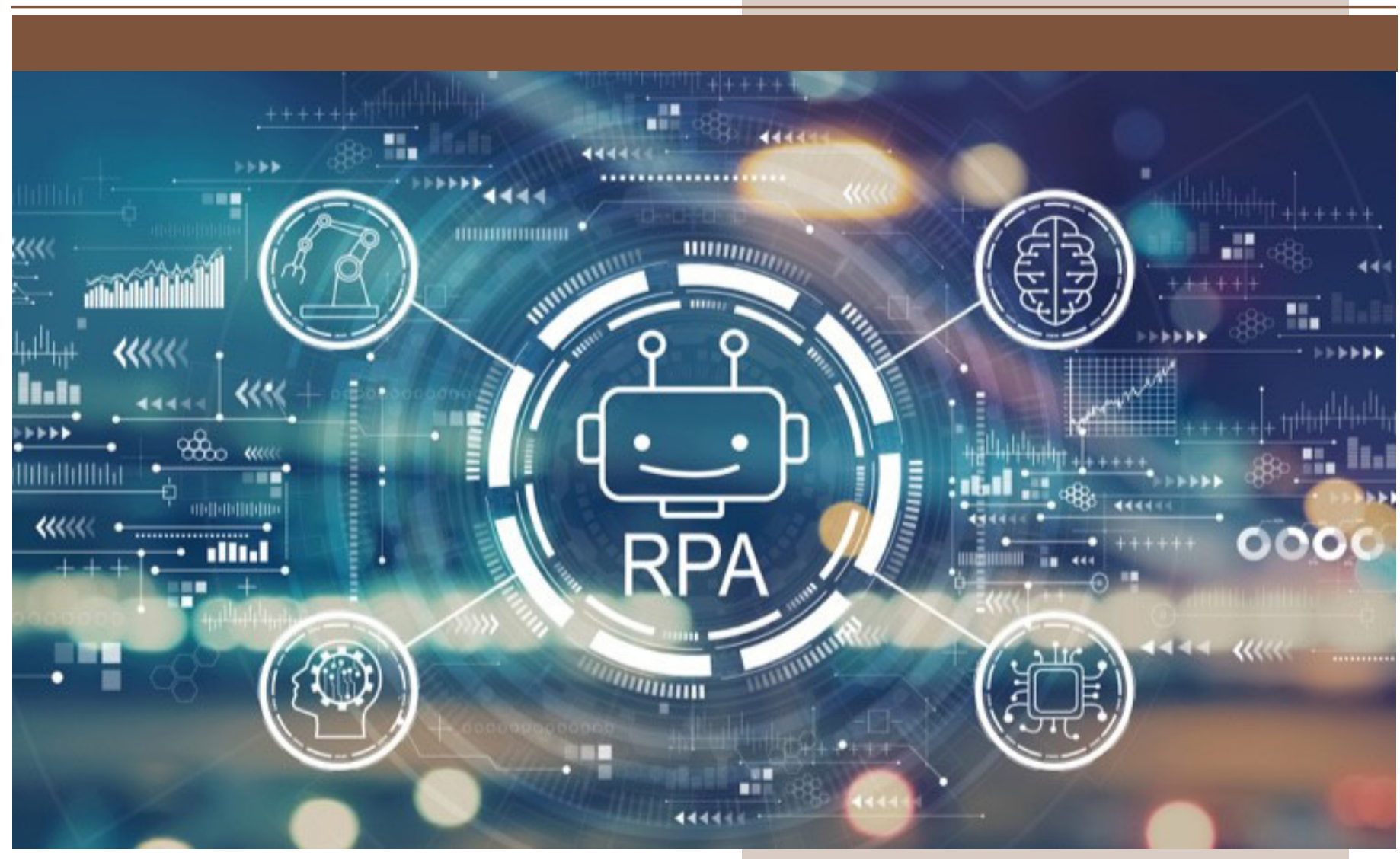

finance department. In contrast, most other new technologies are mainly embedded and aligned into the IT department.

RPA's institutionalisation has to be associated with a holistic change management approach, with proper and structured alignments with organisational employees, RPA implementation team, business processes, legacy systems and existing infrastructure, and the organisational structure and strategic alignment. The scaling up of the RPA implementation from a PoC stage to an organisation-wide stage is the enterprise RPA. Enterprise RPA is built on multiple facets such as bot development, maintenance, security and risk management, software quality assurance, deployment and scalability. Each of these facets has its unique challenges to overcome in the enterprise RPA journey. However, most organisations' key challenge is aligning the RPA rollout to the organisational people, process, and structure [3].

\section{Challenges to overcome in RPA}

\section{deployment and scalability}

When embedding Robotic Process Automation to organisations, there are two key issues. Separation

\section{"RPA has} already proven its value and is expected to be adopted and deployed well into the future"

of business and automation responsibilities, and having an enterprise-wide approach and support are the key challenges faced by organisations when deploying and scaling RPA.

1. Separation of business and automation responsibilities

RPA implementations are typically initiated and governed by business areas with limit- 
ed IT department involvement, unlike traditional IT solutions. However, scaling up towards enterprise RPA is a journey that needs to go hand in hand with business areas, IT and finance departments.

When scaling up beyond the proof-of-concept stage, it is necessary to integrate IT knowledge and other facets such as governance, project management, risk management, quality assurance. Even though the deployment of RPA is relatively easy and quicker compared to other technologies, RPA bot licensing is quite expensive. Therefore, it is essential to identify the right business processes to be automated. To ensure the bots are properly utilised, choosing standardised business processes and reusing the modules during automation is crucial. Business area involvement is vital in understanding and identifying the right processes to automate, modelling the processes to identify the repeatable and reusable tasks in some aspects. Hence, business knowledge is important in setting up the long-term vision for RPA in an organisation. The finance department should also be involved as they dictate the expenses for the technology and the return on investment. Therefore, the governance of RPA technology should be shared between finance, business and IT departments in organisations, which contradicts the existing organisational structure when introducing new technologies. The need for ongoing business, IT and finance alignment challenges the enterprise-wide deployment of RPA and calls for new alignment approaches [4].

\section{Enterprise-wide approach and support}

Unlike traditional and heavyweight IT solutions, RPA does not impact organisational legacy systems and existing IT infrastructure. As a lightweight and more flexible rapid automation technology, RPA has less risk in ad-hoc automation solutions with no end-to-end improvements in business processes.

However, this also means that RPA can be implemented on a smaller scale within one department in one business process, communicating within that specific department and creating a local impact. Therefore, the other departments in organisations would have limited visibility on the technol- ogy and its value, which might hinder the enterprise-wide adoption of RPA. This is another challenge that needs to be addressed by proper change management and institutionalisation of RPA.

\section{Where to next?}

RPA is moving towards hyper-automation by marrying other technologies such as Optical Character Recognition, artificial intelligence, machine learning and chatbot integrations. This will enable the organisations to automate more complex and less rule-based tasks and handle exceptions that currently need human interventions. Smart RPA is also predicted to be cognitive. The bots will learn by acquiring information, reason using the context and rules, and self-correcting through their learning experiences.

\section{References}

[1] C. Boulton, "What is RPA? A revolution in business process automation," Computerworld Hong Kong, 2017 Nov 17 2017-11-23 2017.

[2] Forrester Consulting, "The New Frontier Of Automation: Enterprise RPA.," Forrester Consulting Thought Leadership Paper Commissioned by UiPath.2017.

[3] S. Burnett, M. Aggarwal, A. Modi, and S. Bhadola, "Defining Enterprise RPA.," Everest Group Research.2018.

[4] S. Anagnoste, "Robotic Automation Process-The operating system for the digital enterprise," in Proceedings of the International Conference on Business Excellence, 2018, pp. 54-69.

\section{Article by}

Imesha Vitharanage?, Amila Thibbotuwawa ${ }^{2}$ ${ }^{1}$ Information Systems School, Queensland University of Technology, Australia. ${ }^{2}$ Department of Transport and Logistics Management, Faculty of Engineering, University of Moratuwa, Sri Lanka 